\title{
A simple adjustment of runtimes between stations for saving traction energy by means of mathematical programming
}

\author{
M. Miyatake, R. Kuwahara \& S. Nakasa \\ Sophia University, Japan
}

\begin{abstract}
The authors proposed a comprehensive mathematical formulation as a linear/nonlinear programme for considering energy-saving train scheduling. The formulation is to optimize running time for each section between stations with fixing total time between origin and destination of a train. The original fundamental manner of optimization named "identical incremental energy consumption" was introduced. The model was extended and applied to a simple railway line model with six stations and five interstations. It could be solved with a general-purpose optimization library on a PC. The result showed that optimal condition and energy saving effect could be evaluated easily. Several percent of energy was saved by the optimization process.
\end{abstract}

Keywords: saving energy, train scheduling, optimization.

\section{Introduction}

While railway system is a green transportation, many attempts to reduce energy consumption has been recently seen. Especially in Japanese railways, energy saving and peak power shaving has been encouraged since electrical energy shortage problem arose from the East Japan Disaster on $11^{\text {th }}$ March 2011. Japanese railway operators have experienced that serious shortage of electricity makes train operation difficult.

The authors have been developing the strategy for reducing traction energy by combining the following frameworks;

- saving energy in each interstation by refining train speed profile,

- saving total energy in every interstations by refining train schedule. 
The first item was already reported [1-3] and a few more papers have appeared $[4,5]$. As the second item above, synchronization of an accelerating train and a braking train is one of the common methodology for regenerative energy utilization [6-8]. It is very effective if the number of stations is small, however, it is not always easy to synchronize trains for long lines with many stations and robustness against delay of train departure is suspicious. Therefore, the authors do not consider the synchronization of trains and aim at a simple formulation.

In this paper, the authors propose a comprehensive mathematical formulation as a linear/nonlinear programming for considering energy-saving train scheduling. A similar study can be seen in [9] for high speed trains, however, there are no papers applicable to commuting train routes with many stations.

The proposed formulation is to optimize running time of each section between stations with fixing total time between origin and destination of a train. The original fundamental rule of optimization is firstly introduced as a basis of the method. The rule is to be extended to apply it to various practical conditions. Finally the method is demonstrated in some cases.

\section{Law of "identical incremental energy consumption"}

The relation between runtime $T$ and energy consumption $W$ in the $i$-th interstation is derived as Figure 1. The function $W(T)$ can be derived from simulations as well as measurements. In this paper, $W(T)$ is assumed as convex downward. The minimum point of $T$ of the $W(T)$ graph is the flat-out time that is achieved with maximum acceleration/deceleration and without coasting. In the scheduling phase, the fraction of the flat-out time is often raised to the regular runtime which is given as discretized numbers.

The sum of runtimes $T_{S}$ throughout whole interstations is fixed. In order to minimize the total energy consumption in running whole interstations, the

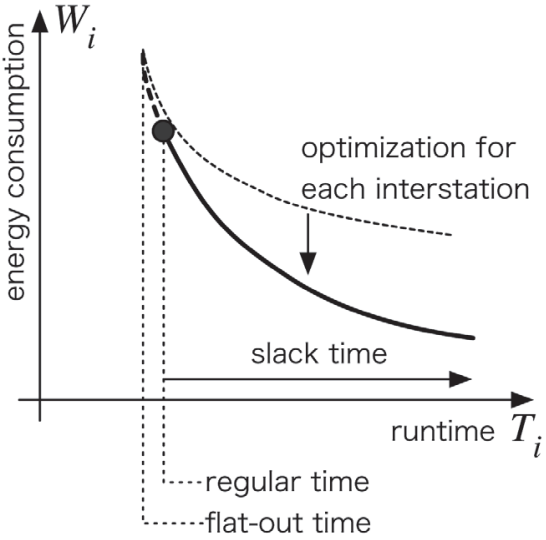

Figure 1: Relation between runtime and energy consumption.

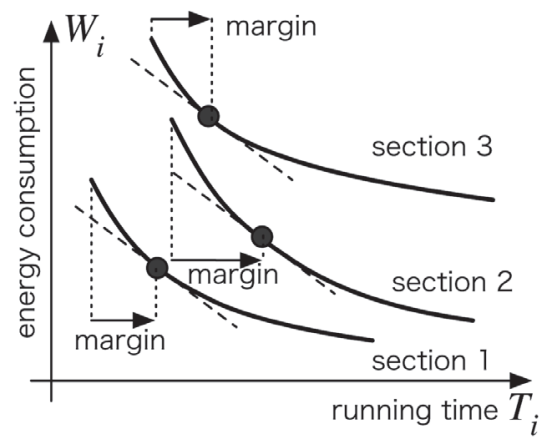

Figure 2: The principle of incremental energy consumption. 
following nonlinear optimization problem with a linearity constraint is solved. In this study, dwell time at each station is unchanged.

$$
\begin{gathered}
J\left(T_{1}, \cdots, T_{N}\right)=\sum_{i=1}^{N} W_{i}\left(T_{i}\right) \rightarrow \min \\
\text { subject to } \quad \sum_{i=1}^{N} T_{i}=T_{S}
\end{gathered}
$$

The following optimal condition is derived from applying Lagrange multiplier technique.

$$
\frac{\partial W_{1}}{\partial T_{1}}=\frac{\partial W_{2}}{\partial T_{2}}=\cdots=\frac{\partial W_{N}}{\partial T_{N}}
$$

One can easily find a simple law of minimizing the energy consumption that every runtimes are optimal if the incremental energy consumptions are identical as illustrated in Figure 2. This is named as law of "identical incremental energy consumption" [10]. If the actual train schedule does not satisfy the law, the interstation with large $|\partial W / \partial T|$ should increase the runtime and vice versa.

\section{Generalized model for considering practical conditions}

\subsection{Expansion of the model}

If minimum and maximum runtimes are given from passengers' point of view etc., the runtime $T_{i}$ should be in $\left[T_{i}^{\min }, T_{i}^{\max }\right]$, that is;

$$
T_{i}^{\min } \leq T_{i} \leq T_{i}^{\max }
$$

Here, groups of interstations for providing time constraints are defined while only sum of runtimes was provided for whole operating line in section 2 . When the $j$-th group of interstations $s_{j} \subseteq\{1,2, \cdots, N\}$ is defined, sum of the runtimes for $s_{j}, \sum_{i \in s_{j}} T_{i}$, will be in $\left[T_{S_{-j}}^{\min }, T_{S_{-} j}^{\max }\right]$,

$$
T_{S_{-j}}^{\min } \leq \sum_{i \in s_{j}} T_{i} \leq T_{S_{-j}}^{\max }
$$

The key for successful analyses is to find the appropriate function $W(T)$, however, it is generally difficult. It is inevitable to treat the relation between $W$ and $T$ as an implicit function for more precise curve-fitting against various conditions as

$$
f_{i}\left(T_{i}, W_{i}\right)=0 .
$$

After this, $W$ is re-defined as a variable. 


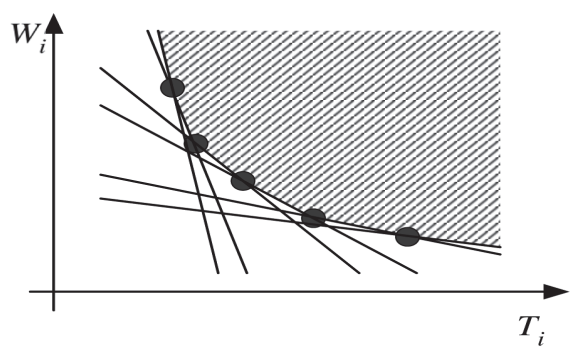

Figure 3: Linear approximation of nonlinear $W-T$ characteristics.

The original problem explained in section 2 is expanded as (7)-(10) by regarding (6) as a new constraint,

$$
\begin{aligned}
J\left(T_{1}, \cdots, T_{N}, W_{1}, \cdots, W_{N}\right)= & \sum_{i=1}^{N} W_{i} \rightarrow \min \\
\text { subject to } \quad & T_{S_{-j}}^{\min } \leq \sum_{i \in s_{j}} T_{i} \leq T_{S_{-j}}^{\max }(j=1, \cdots, M), \\
& T_{i}^{\min } \leq T_{i} \leq T_{i}^{\max } \quad(i=1, \cdots, N) \\
& f_{i}\left(T_{i}, W_{i}\right) \leq 0 \quad(i=1, \cdots, N)
\end{aligned}
$$

where $M$ is the number of groups.

In (10), the implicit function $f$ is defined so as to satisfy $f_{i}(0,0)>0$, then the linearity constraint (6) was relaxed to a nonlinearity one. This relaxation has another advantage that any nonlinear convex constraints of (10) can be approximated with some linear constraints as illustrated in Figure 3. It means that data of $W-T$ characteristics can be directly included into the constraints without assuming type of function $f_{i}\left(T_{i}, W_{i}\right)$. Finally, since all the constraints becomes linear, the problems can be solved with linear programming.

\subsection{Generalized model as a linear/nonlinear optimization problem}

The following vectors are defined in order to generalize the equations.

$$
\begin{aligned}
& \boldsymbol{T}=\left[\begin{array}{lll}
T_{1} & \cdots & T_{N}
\end{array}\right]^{T}, \\
& \boldsymbol{W}=\left[\begin{array}{lll}
W_{1} & \cdots & W_{N}
\end{array}\right]^{T}, \\
& \boldsymbol{f}(\boldsymbol{T}, \boldsymbol{W})=\left[f_{1}(\boldsymbol{T}, \boldsymbol{W}) \cdots f_{N}(\boldsymbol{T}, \boldsymbol{W})\right]^{T}, \\
& \boldsymbol{T}_{S}^{\min }=\left[\begin{array}{lll}
T_{S_{-} 1}^{\min } & \cdots & T_{S_{-} M}^{\min }
\end{array}\right]^{T} \text {, } \\
& \boldsymbol{T}_{S}^{\max }=\left[\begin{array}{lll}
T_{S_{-} 1}^{\max } & \cdots & T_{S_{-} M}^{\max }
\end{array}\right]^{T}, \\
& \boldsymbol{T}^{\min }=\left[\begin{array}{lll}
T_{1}^{\min } & \cdots & T_{N}^{\min }
\end{array}\right]^{T}, \\
& \boldsymbol{T}^{\max }=\left[\begin{array}{lll}
T_{1}^{\max } & \cdots & T_{N}^{\max }
\end{array}\right]^{T}, \\
& \boldsymbol{c}=\left[\begin{array}{llll}
1 & 1 & \cdots & 1
\end{array}\right] \text {. }
\end{aligned}
$$


The superscript letter $T$ means transposing, $f(T, W)$ is defined as a vector function. If linear approximation will be applied to $f(T, W)$, dimension of the $\boldsymbol{f}(\boldsymbol{T}, \boldsymbol{W})$ is much higher than $N$.

By using these vectors (7)-(10) are transformed to (19)-(22),

$$
\begin{aligned}
J(\boldsymbol{T}, \boldsymbol{W})= & J(\boldsymbol{W})=\boldsymbol{c} \boldsymbol{W} \rightarrow \min , \\
\text { subject to } \quad & \boldsymbol{T}_{S}^{\min } \leq \boldsymbol{A} \boldsymbol{T} \leq \boldsymbol{T}_{S}^{\max } \\
& \boldsymbol{T}^{\min } \leq \boldsymbol{T} \leq \boldsymbol{T}^{\max } \\
& \boldsymbol{f}(\boldsymbol{T}, \boldsymbol{W}) \leq \mathbf{0} .
\end{aligned}
$$

The matrix $\boldsymbol{A}$ defines the groups of interstations $s_{j}$. If the interstation $i$ is included in $s_{j}$, the $i$-th column of $j$-th row is 1 , otherwise it is 0 . In the example of case 3 shown in the next section, $\boldsymbol{A}$ is composed as

$$
\boldsymbol{A}=\left[\begin{array}{llllllllll}
1 & 1 & 1 & 1 & 1 & 1 & 1 & 1 & 1 & 1 \\
1 & 1 & 0 & 0 & 0 & 0 & 0 & 0 & 0 & 0 \\
0 & 0 & 0 & 0 & 0 & 0 & 0 & 0 & 1 & 1
\end{array}\right]
$$

\subsection{Solving mathematical model}

The generalized problem as (19)-(22) can be solved with a typical Nonlinear Programming (NLP). Since (22) is the only nonlinear constraint, the linearization of (22) will make the problem to solve with Linear Programming (LP). In any cases, various general-purpose mathematical libraries can be applied to the problem. In this study, "Optimization Toolbox" of the MATLAB was used.

\section{Demonstration of availability of the model}

The proposed model was applied to a virtual railway line with six stations and five interstations for demonstration as illustrated in Figure 4. The example was intended to minimize the energy consumption of a train in round-trip operation by adjusting from $T_{1}$ to $T_{10}$.

Fundamental specifications of a 10 car train, a typical commuter train in Japan, were the same as that in [10]. The relation between $T_{i}$ and $W_{i}$ plotted in

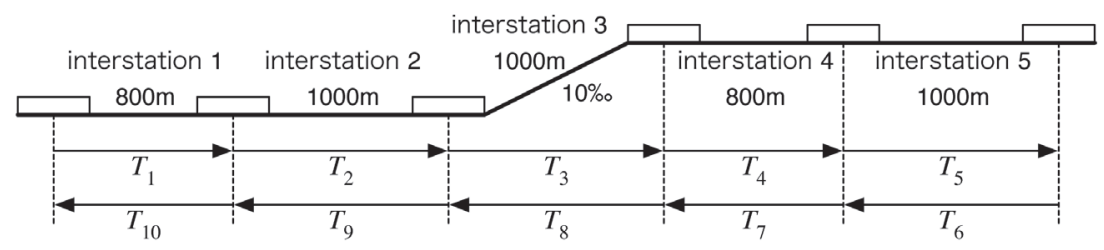

Figure 4: Assumed track condition for simulation. 


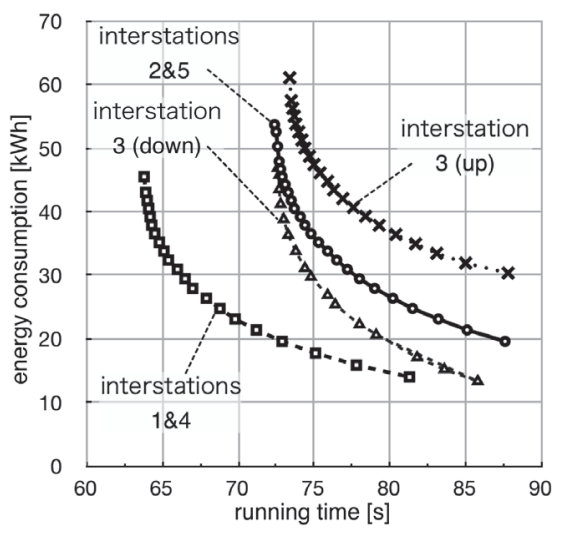

(a) without regenerative braking

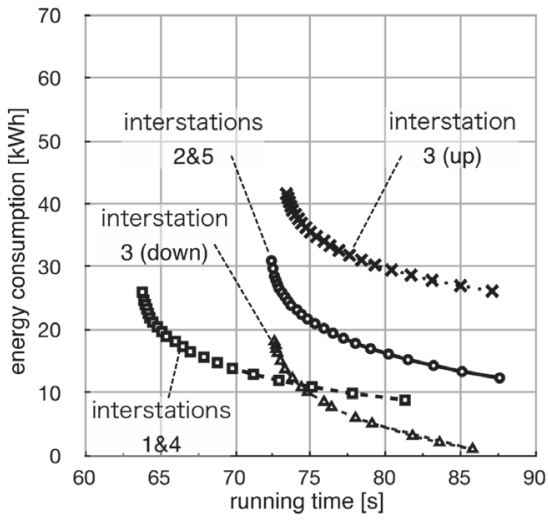

(b) with regenerative braking

Figure 5: Calculated relation between runtime and energy consumption.

Figure 5 was calculated for each interstation with assuming the profile composed of maximum acceleration, coasting and deceleration at $80 \%$ of the maximum value. Each regular runtime is set as tabulated in Table 2.

Approximated function $f\left(T_{i}, W_{i}\right)$ was derived from Figure 5. The following implicit function was fitted to the curve by trial and error.

$$
f\left(T_{i}, W_{i}\right)=a_{3} W_{i}^{3}+a_{2} W_{i}^{2}+a_{1} W_{i}+a_{0}-T_{i}=0 .
$$

The coefficients for each case are tabulated in Table 1 . Since the coefficients of determination of the regression for every cases were 0.998 and more in the curve fitting, the approximation of (24) was quite good.

Three cases with different constraints as Tables 2 and 3 were assumed:

Case 1 is a conventional slack time distribution.

Case 2 is the optimal case.

Case 3 is also the optimal case but two more groups of interstations are added for faster runtimes in interstations $1,2,9$ and 10 .

The results of optimal slack times are shown in Figures 6 and 7. Energy consumption for each case is tabulated in Table 4 . The results were considered from the following points of view.

1. Influence of regenerative braking on solutions

With comparing Figure 6(a) and (b), very few difference of the optimal slack times are found. The same phenomenon is seen in Figure 7. Although energy consumption shown in Table 4 is quite large, there are very few influence of regenerative braking on these solutions.

2. Influence of linearization of $W-T$ characteristic on solutions

Figures 6 and 7 indicate that the linearization at every one second causes round-up/down of the slack times. However, there are very few difference 
Table 1: Curve-fitting of $T_{i}-W_{i}$ characteristics.

(a) without regenerative braking

\begin{tabular}{|c||c|c|c|c|}
\hline$i$ & $1,4,7,10$ & $2,5,6,9$ & 3 & 8 \\
\hline \hline$a_{3}\left[\times 10^{-4}\right]$ & -7.6752 & -4.3543 & -6.5680 & -3.4770 \\
\hline$a_{2}\left[\times 10^{-2}\right]$ & 9.2938 & 6.5556 & 11.058 & 4.7287 \\
\hline$a_{1}$ & -3.8506 & -3.3644 & -6.2958 & -2.1928 \\
\hline$a_{0}$ & 118.68 & 131.39 & 194.84 & 107.27 \\
\hline
\end{tabular}

(b) with regenerative braking

\begin{tabular}{|c||c|c|c|c|}
\hline$i$ & $1,4,7,10$ & $2,5,6,9$ & 3 & 8 \\
\hline \hline$a_{3}\left[\times 10^{-4}\right]$ & -45.870 & -25.630 & -37.192 & -22.507 \\
\hline$a_{2}\left[\times 10^{-2}\right]$ & 32.120 & 22.469 & 44.847 & 12.014 \\
\hline$a_{1}$ & -7.6838 & -6.7122 & -18.235 & -2.2823 \\
\hline$a_{0}$ & 126.86 & 140.78 & 323.55 & 87.857 \\
\hline
\end{tabular}

Table 2: Regular runtimes and assumed added slack times in case 1.

\begin{tabular}{|c||c|c|c|c|c|c|c|c|c|c|}
\hline Cases & $\begin{array}{c}T_{1} \\
{[\mathrm{~s}]}\end{array}$ & $\begin{array}{c}T_{2} \\
{[\mathrm{~s}]}\end{array}$ & $\begin{array}{c}T_{3} \\
{[\mathrm{~s}]}\end{array}$ & $\begin{array}{c}T_{4} \\
{[\mathrm{~s}]}\end{array}$ & $\begin{array}{c}T_{5} \\
{[\mathrm{~s}]}\end{array}$ & $\begin{array}{c}T_{6} \\
{[\mathrm{~s}]}\end{array}$ & $\begin{array}{c}T_{7} \\
{[\mathrm{~s}]}\end{array}$ & $\begin{array}{c}T_{8} \\
{[\mathrm{~s}]}\end{array}$ & $\begin{array}{c}T_{9} \\
{[\mathrm{~s}]}\end{array}$ & $\begin{array}{c}T_{10} \\
{[\mathrm{~s}]}\end{array}$ \\
\hline \hline regular & 65 & 75 & 75 & 65 & 75 & 75 & 65 & 75 & 75 & 65 \\
\hline 1 & 0 & 5 & 5 & 5 & 5 & 0 & 5 & 5 & 5 & 5 \\
\hline
\end{tabular}

Table 3: Constraints for cases 2 and 3.

\begin{tabular}{|c||c|c|c|c|c|}
\hline Cases & $T_{1,4,7,10}[\mathrm{~s}]$ & $T_{2,3,5,6,8,9}[\mathrm{~s}]$ & $\sum_{i=1}^{10} T_{i}[\mathrm{~s}]$ & $\sum_{i=1}^{2} T_{i}[\mathrm{~s}]$ & $\sum_{i=9}^{10} T_{i}[\mathrm{~s}]$ \\
\hline \hline 2 & $65 \sim 75$ & $75 \sim 85$ & $720 \sim 750$ & - & - \\
\hline 3 & $65 \sim 75$ & $75 \sim 85$ & $720 \sim 750$ & $140 \sim 145$ & $140 \sim 145$ \\
\hline
\end{tabular}

in energy consumption from Table 4 . Therefore, such fine linearization is a good technique to make modeling easy and reduce computation time.

3. Comparison among three cases

With comparing energy consumption of the cases 1 and 2 in Table 4, 2\% and more energy was saved by the optimization. In case 3 with two more constraints, consumed energy was slightly increased from case 2 by less than $1 \%$. Under the runtime optimization framework, energy consumption could be kept still smaller than that in non-optimization.

In Table 4, energy consumption in running with regular runtimes was also shown. Since much more energy was consumed than the cases 1-3, keeping long runtimes is the key to reduce energy consumption. 
Table 4: Total energy consumption.

\begin{tabular}{|c||c|l|l|l|}
\hline \multicolumn{1}{|c||}{} & \multicolumn{4}{c|}{ total energy [kWh] } \\
\hline \multicolumn{1}{|c||}{} & without regenerative braking & \multicolumn{2}{c|}{ with regenerative braking } \\
\hline cases & NLP & LP & NLP & LP \\
\hline \hline 1 & \multicolumn{2}{c|}{275.21} & \multicolumn{2}{c|}{165.44} \\
\hline 2 & 268.29 & 268.31 & 161.69 & 161.70 \\
\hline 3 & 269.72 & 269.77 & 162.45 & 162.48 \\
\hline \hline regular time & \multicolumn{3}{c|}{355.60} & \multicolumn{2}{c|}{209.86} \\
\hline
\end{tabular}

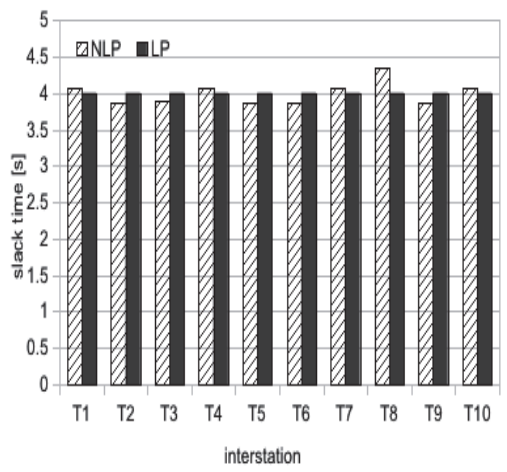

(a) without regenerative energy

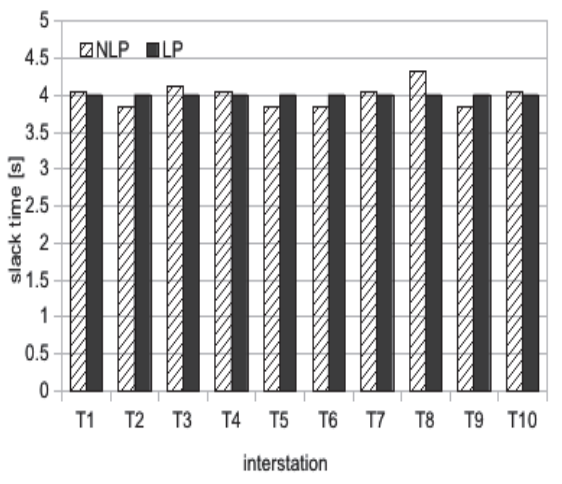

(b) with regenerative energy

Figure 6: Optimal slack times for case 2.

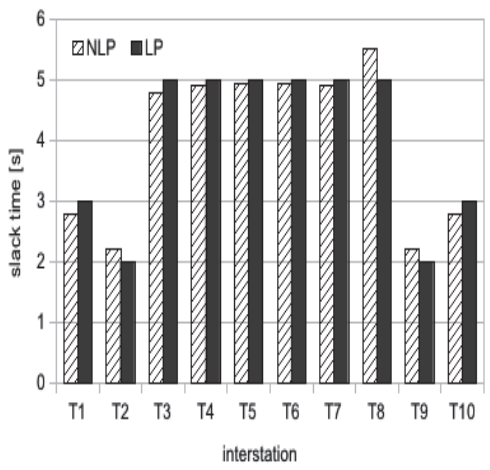

(a) without regenerative energy

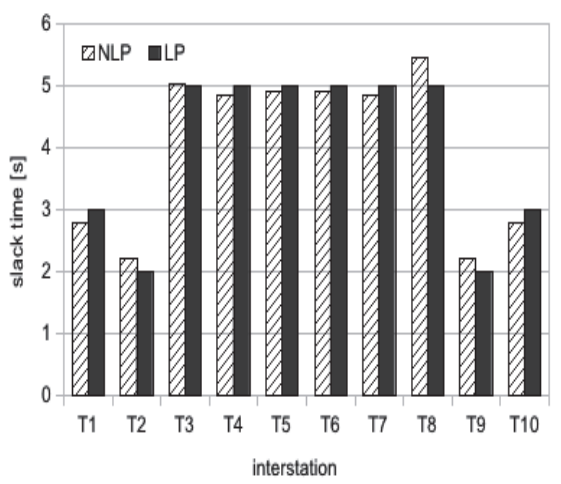

(b) with regenerative energy

Figure 7: Optimal slack times for case 3.

\section{Conclusion}

In this paper, a general law of minimal condition named "identical incremental energy consumption" was introduced. The concept was expanded in order to 
consider some practical constraints. The proposals were demonstrated through simulation. The result showed that optimal slack time distribution and energy saving effect could be evaluated easily. At least several percent of energy was saved by the optimization process.

This method is quite effective in applying to the train with large slack time. However, it should be noted that adjusting schedule may affect utility of passengers and robustness of operation against train delay. The authors will consider them as well as energy consumption in order to manage the slack times more properly.

\section{References}

[1] H. Ko, T.Koseki and M. Miyatake: "Application of Dynamic Programming to Optimization of Running Profile of A Train", Computers in Railways IX, WIT Press, pp. 103-112, 2004.

[2] H. Ko and M. Miyatake "A numerical Algorithm for Run-curve Optimization of Trains Considering a DC Feeding Circuit" Computers in Railways X, WIT Press, pp. 787-796, 2006.

[3] M. Miyatake and H. Ko: "Optimization of Train Speed Profile for Minimum Energy Consumption" IEEJ Transactions on Electrical and Electronics Engineering, Vol.5, No.3, pp. 263-269, 2010.

[4] T. Katori and T. Izumi: "A Production Train Diagram of Train Control to Save Power Consumption Used for Dynamic Programming" Computers in Railways XI, WIT Press, pp. 359-368, 2008.

[5] M. Dominguez, A. Fernandez, A.P. Cucala and L.P. Cayuela: "Computeraided Design of ATO Speed Commands According to Energy Consumption Criteria” Computers in Railways XI, WIT Press, pp. 183-192, 2008.

[6] H.J. Guo, H. Ohashi and O. Ichinokura : "DC Electric Train Traffic Scheduling Method Considering Energy-Saving" IEEJ Transactions on Industry Applications, 119-D-11, pp. 1337-1344, 1999. (in Japanese)

[7] T. Albrecht: "Reducing Power Peaks and Energy Consumption in Rail Transit Systems by Simultaneous Train Running Time Control" Computers in Railways IX, WIT Press, pp. 885-894, 2004.

[8] K.M. Kim, K.T. Kim and M.S. Han : "A Model and Approaches for Synchronized Energy Saving in Timetable" Proceedings of WCRR 2011, No. A4-1, 2011.

[9] C. Sicre, P. Cucala, A. Fernandez, J. A. Jimenez, I. Ribera and A. Serrano : “A Method to Optimise Train Energy Consumption Combining Manual Energy Efficient Driving and Scheduling" Computers in Railways XII, WIT Press, pp. 549-560, 2010.

[10] M. Miyatake: “A Simple Mathematical Model for Energy-saving Train Scheduling" IEEJ Transactions on Industry Applications, 131-D-6, 2011 (in Japanese). 